\title{
Correction to: Lederbergia citri sp. nov., and Lederbergia citrisecundus sp. nov., isolated from citrus rhizosphere
}

\author{
Guo-Hong Liu ${ }^{1} \cdot$ Manik Prabhu Narsing Rao ${ }^{2}$ Q Qian-Qian Chen ${ }^{1}$ Jian-Mei Che ${ }^{1} \cdot$ Huai Shi $^{1} \cdot$ Bo Liu $^{1} \cdot$ Wen-Jun Li $^{2}$
}

Published online: 17 February 2022

(c) Springer Science+Business Media, LLC, part of Springer Nature 2022

\section{Correction to: Current Microbiology https://doi.org/10.1007/s00284-021-02741-0}

The original version of this article unfortunately contained a mistake. The authors would like to correct species name "Lederbergia citrisecundus". It was brought to our attention that the proposed name Lederbergia citrisecundus was incorrect as it contravenes Rule 12c(1) of the International Code of Nomenclature of Prokaryotes (ICNP). Although we acknowledged Prof. Aharon Oren (The Alexander Silberman Institute of Life Sciences, The Hebrew University of Jerusalem, Israel) for nomenclature help, his name was indicated erroneously and he cannot be made responsible for the wrong name and epithet. This is entirely the author's mistake. We, therefore, propose changing the name of the newly proposed species to Lederbergia citrisecunda as follows:

\section{Description of Lederbergia citrisecunda sp. nov.}

Lederbergia citrisecunda (ci.tri.se.cun'da. L. fem. n. citrus, the citrus tree; L. num. ord. adj. secundus, the second; N.L. fem. adj. citrisecunda, the second isolated from the citrus

The original article can be found online at https://doi.org/10.1007/ s00284-021-02741-0.

\footnotetext{
Bo Liu

fzliubo@163.com

$\triangle$ Wen-Jun Li

liwenjun3@mail.sysu.edu.cn

1 Agricultural Bio-Resources Institute, Fujian

Academy of Agricultural Sciences, Fujian 350003,

People's Republic of China

2 State Key Laboratory of Biocontrol, Guangdong Provincial, Key Laboratory of Plant Resources and Southern Marine Science and Engineering Guangdong Laboratory (Zhuhai), School of Life Sciences, Sun Yat-Sen University, Guangzhou 510275, People's Republic of China
}

tree). Cells are Gram-positive, aerobic and motile. Colonies are circular, flat and rough. Elliptic endospore is located in the center. Growth occurs at $20-45{ }^{\circ} \mathrm{C}$ (optimum 35-40 ${ }^{\circ} \mathrm{C}$ ) and $\mathrm{pH} 6.0-10.0$ (optimum $\mathrm{pH} 8.0$ ) with $0-5 \%(\mathrm{w} / \mathrm{v})$ $\mathrm{NaCl}$ (optimum 2\%). Catalase and oxidase are positive. Acid is produced from amygdalin, esculin, saligenin, cellobiose, maltose, lactose, D-melibiose, sucrose, trehalose, gentiobiose and D-turanose. In API ZYM system, positive for alkaline phosphatase, esterase (C4), acid phosphatase, naphthol-AS-BI-phosphohydrolase, $\beta$-galactosidase, and $\alpha$-galactosidase. The cell-wall peptidoglycan contains mesodiaminopimelic acid. MK-7 is the only isoprenoid quinone present. The major fatty acids are anteiso- $\mathrm{C}_{15: 0}$ and iso- $\mathrm{C}_{15: 0}$. The polar lipids are diphosphatidylglycerol, phosphatidylethanolamine, phosphatidylglycerol, unidentified glycolipid, unidentified aminolipid and unidentified phospholipid. The genomic DNA G $+\mathrm{C}$ content is $36.7 \%$. The type strain FJAT$49732^{\mathrm{T}}\left(=\right.$ CCTCC AB $2019246^{\mathrm{T}}=$ LMG $31584^{\mathrm{T}}$ ), was isolated from the rhizosphere of the citrus plant. The $16 \mathrm{~S}$ rRNA gene and genome sequences of strain FJAT-49732 ${ }^{\mathrm{T}}$ have been deposited in the GenBank/ENA/DDBJ under the accession numbers MZ144014 and JAGYPJ000000000, respectively.

Further information on the properties of the type strain FJAT- $49732^{\mathrm{T}}$ is given in the original version of the article (https://doi.org/10.1007/s00284-021-02741-0).

Publisher's Note Springer Nature remains neutral with regard to jurisdictional claims in published maps and institutional affiliations. 